\title{
EDITORIAL
}

\section{BRAIN INJURY IN PRETERM NEWBORN INFANTS}

\author{
Mário Cícero Falcão
}

RHCFAP/2979

FALCÃO, M.C. et al. - Brain injury in preterm newborn infants. Rev. Hosp. Clín. Fac. Med. S. Paulo 54 (5):139-140,1999.

Brain injury in the premature infant is an extremely important problem, in part because of the large number of infants affected yearly.

The magnitude of this problem in the preterm newborn and, in particular, the importance of prevention of this injury is enormous.

Early studies identified intracranial hemorrhage as being responsible for most of the severe brain damage noted in preterm infants. The proposed pathogenic mechanisms implicated complications commonly associated with respiratory distress, including hypoxemia, ischemia, and blood pressure fluctuation.

The predisposing conditions for development of intracranial hemorrhage may be categorized under intravascular inflow, intravascular outflow, and structural factors. Before term, the vasculature of the germinal matrix region lacks musculorum, is poorly supported by perivascular structures, and is an end bed to the early muscularized arterioles in the cerebrum. Loss of vascular autoregulation in these arterioles, leading to a pressure-passive state, makes the vessels vulnerable to rupture during fluctuations in arterial blood flow, primarily when venous pressure increases.

The intravascular factors include events that provoke surges in cerebral inflow or compromise the outflow of blood on the venous side in the circulation matrix. Surges of cerebral blood flow may occur with seizures, hypoxia, apnea, respiratory distress, patent ductus arterious, extracorporeal membrane oxygenation, and certain care-taking procedures such as tracheal suctioning. Increased venous pressure may be associated with respiratory distress syndrome, pneumothorax, congestive heart failure, high continuous positive airway pressure, and possibly hyperviscosity.

The contribution to brain injury in preterm newborns by postnatal events remains to be elucidated. Despite many studies, the threshold of hypoxemia or ischemia below which periventricular leukomalacia or intracranial hemorrhage occurs remains unknown. Thus pathophysiologic mechanisms responsible for periventricular leukomalacia and intracranial hemorrhage associated with prematurity are incompletely defined. Therefore, at the present time the measures that should be taken postnatally to prevent brain injury in preterm infants are poorly understood.

The prevention of cerebral ischemia is critical for prevention of

From the Department of Pediatrics, University of São Paulo School of Medicine, São Paulo Brazil. preventricular leukomalacia. Important factors for the prevention of cerebral ischemia include: careful attention to blood pressure to avoid systemic hypotension; careful attention to blood gases to avoid severe hypocarbia; avoidance of rapid intravenous administration of osmotically active agents; avoidance of unnecessary manipulations of the infants; detection of pressure-passive cerebral circulation and stabilization of the cerebral circulation in infants with this circulatory abnormality (note that the means for stabilization of pressure-passive circulation depends on the cause).

Several pharmaceutical compounds have been studied as possible treatments for prevention of brain damage in premature newborns. In studies to date, some compounds have produced positive - though not conclusive results, some equivocal results, and others have not proved beneficial.

Phenobarbital does not prevent the development of intraventricular hemorrhage. The use of indomethacin as a prophylactic agent against the intraventricular hemorrhage remains controversial and requires further clinical studies prior to recommendations for its routine use.

The prophylactic use of pancuronium has been reported to reduce the risk of intraventricular hemorrhage in 
specific preselected populations; however, studies are needed to affirm the beneficial effects reported in the initial studies.

Prenatal treatment of the fetus with steroids to prevent respiratory distress syndrome could decrease the incidence of intraventricular hemorrhage.
Recent epidemiologic studies suggest that intrapartum magnesium sulfate exposure is followed by a markedly decrease risk for subsequent cerebral palsy in very low-birth weight infants. However, this apparent beneficial effect has not been established conclusively.
Researchers have progressed in their understanding of the pathogenesis, prevention, and treatment of the important problem of brain injury in premature infants, but sustantial additional research is needed for the development and testing of effective treatment and preventative methodologies. 